\title{
A description and qualitative comparison of the elements of heterogeneous bovine viral diarrhea control programs that influence confidence of freedom
}

\author{
A. M. van Roon, ${ }^{1 *}$ I. M. G. A. Santman-Berends, ${ }^{1,2}$ D. Graham, ${ }^{3}$ S. J. More, ${ }^{4}$ M. Nielen, ${ }^{1}$ L. van Duijn, ${ }^{2}$ \\ M. Mercat, ${ }^{5}$ C. Fourichon, ${ }^{5}$ A. Madouasse, ${ }^{5}$ J. Gethmann, ${ }^{6}$ C. Sauter-Louis, ${ }^{6}$ J. Frössling, ${ }^{7}$ A. Lindberg, ${ }^{7}$ \\ C. Correia-Gomes, ${ }^{8}$ G. J. Gunn, ${ }^{8}$ M. K. Henry, ${ }^{8}$ and G. van Schaik ${ }^{1,2}$ \\ ${ }^{1}$ Department of Farm Animal Health, Faculty of Veterinary Medicine, Utrecht University, PO Box 80151, 3508, TD Utrecht, the Netherlands \\ ${ }^{2}$ GD Animal Health, PO Box 9, 7400 AA, Deventer, the Netherlands \\ ${ }^{3}$ Animal Health Ireland, Unit 4/5, The Archways, Bridge St., Carrick-on-Shannon, Co. Leitrim N41 WN27, Ireland \\ ${ }^{4}$ Centre for Veterinary Epidemiology and Risk Analysis, UCD School of Veterinary Medicine, University College Dublin, Belfield, \\ Dublin D04 W6F6, Ireland \\ ${ }^{5}$ BIOEPAR, INRA, Oniris, La Chantrerie, Nantes 44307, France \\ ${ }^{6}$ Institute of Epidemiology, Friedrich-Loeffler-Institute, Südufer 10, 17493 Greifswald, Germany \\ ${ }^{7}$ Department of Disease Control and Epidemiology, National Veterinary Institute (SVA), 75189 Uppsala, Sweden \\ ${ }^{8}$ Scotland's Rural College, Kings Buildings, West Mains Road, Edinburgh, EH9 3JG, United Kingdom
}

\section{ABSTRACT}

For endemic infections in cattle that are not regulated at the European Union level, such as bovine viral diarrhea virus (BVDV), European Member States have implemented control or eradication programs (CEP) tailored to their specific situations. Different methods are used to assign infection-free status in CEP; therefore, the confidence of freedom associated with the "free" status generated by different CEP are difficult to compare, creating problems for the safe trade of cattle between territories. Safe trade would be facilitated with an output-based framework that enables a transparent and standardized comparison of confidence of freedom for CEP across herds, regions, or countries. The current paper represents the first step toward development of such a framework by seeking to describe and qualitatively compare elements of CEP that contribute to confidence of freedom. For this work, BVDV was used as a case study. We qualitatively compared heterogeneous BVDV CEP in 6 European countries: Germany, France, Ireland, the Netherlands, Sweden, and Scotland. Information about BVDV CEP that were in place in 2017 and factors influencing the risk of introduction and transmission of BVDV (the context) were collected using an existing tool, with modifications to collect information about aspects of control and context. For the 6 participating countries, we ranked all individual elements of the CEP and their contexts that could influence the probability that cattle from a herd catego-

Received May 6, 2019.

Accepted January 2, 2020.

*Corresponding author: a.m.vanroon@uu.nl rized as BVDV-free are truly free from infection. Many differences in the context and design of BVDV CEP were found. As examples, CEP were either mandatory or voluntary, resulting in variation in risks from neighboring herds, and risk factors such as cattle density and the number of imported cattle varied greatly between territories. Differences were also found in both testing protocols and definitions of freedom from disease. The observed heterogeneity in both the context and CEP design will create difficulties when comparing different $\mathrm{CEP}$ in terms of confidence of freedom from infection. These results highlight the need for a standardized practical methodology to objectively and quantitatively determine confidence of freedom resulting from different CEP around the world.

Key words: freedom from infection, surveillance, control program, bovine viral diarrhea virus

\section{INTRODUCTION}

Several European member states have implemented control or eradication programs (CEP) tailored to their own specific needs for controlling endemic infections in cattle that are not currently regulated at the European Union (EU) level. Each CEP can apply across an entire member state or over a territory within a member state. These CEP bring tangible benefits to participating farmers and national economies and should be strongly supported by government and other stakeholders. However, substantial differences in CEP create difficulties for intra-community trade. These arise from differences in definitions of infection-free status and the absence of an agreed framework to assess confidence in freedom from infection in cattle moved between countries and regions. 
Within the EU, member states are not allowed to set trade barriers on intra-community trade for cattle diseases that are not regulated at the EU level. This is consistent with the free movement of goods within the EU, a central tenet of the common market, but does pose difficulties with respect to animal disease control. Given this context, it would greatly facilitate safe trade of cattle between member states if there were an objective means by which claims of freedom from infection for all relevant diseases could be evaluated and compared. Currently, however, the CEP can differ substantially, and CEP outputs can be very difficult to compare. In the past, freedom from infection claims were underpinned by defined input standards that provide a detailed description of the activity required, such as testing protocol(s) based on negative test result(s), and these were accepted as proof of freedom from infection (More et al., 2009; Schuppers et al., 2012). However, the probability and associated uncertainty that an animal or herd is truly free from infection is not solely dependent on test result and related test characteristics, but is also influenced by the risk that infection had been introduced into the herd before initial testing but not (yet) detected, or had been (re)introduced into the herd subsequent to testing (or between rounds of testing; Schuppers et al., 2012). This suggests that a more accurate estimation of confidence of freedom from infection can be achieved through an output-based approach, noting that differing sanitary measures have the potential to provide the same level of animal health protection (More et al., 2009). Using this approach, account should be taken of factors that influence the risks of either not detecting infection if present or of introducing infection, such as test procedures preceding export, the geographic location of herds, and animal movements (More et al., 2009; Schuppers et al., 2012; Toftaker et al., 2018).

The STOC free project (Surveillance analysis Tool for Outcome-based Comparison of the confidence of FREEdom from infection) is seeking to fill this key knowledge gap by developing an output-based framework that enables a transparent and standardized comparison of confidence of freedom for CEP across herds, regions, or countries (van Roon et al., 2019; https:/ /www.stocfree.eu/). Ultimately, the project aims to develop simple and practical tools to inform farmers of infection risk when buying animals from certain territories and farms within territories. The project builds on earlier work to evaluate confidence in freedom in CEP, where a range of methods have been used, including scenario-tree analysis and Bayesian and latent class modeling (Martin et al., 2007; Cameron, 2012; Schuppers et al., 2012). This earlier work is promising but has not yet been translated into simple and practical field-based tools.

The current paper represents the first step in the STOC free project and focuses on detailed understanding of those elements of CEP that are relevant to the assessment of confidence in freedom. This information is critical baseline information that will inform later work toward the development of the afore-mentioned output-based framework. For this work, bovine viral diarrhea (BVD; Olafson and Rickard 1947; Houe, 2003) was used as a case study, given the complexity of its infection dynamics and the multiple differences between European member states in terms of infection prevalence, CEP design and implementation (including variation in test methods and sampling schemes), and progress toward control and eradication. Therefore, the confidence of freedom from herds considered negative will not necessarily be equivalent because of variation in context between different territories.

This study sought to describe the elements of CEP that contribute to confidence of freedom - the likelihood that a bovine from a herd categorized as bovine viral diarrhea virus (BVDV)-free is truly free from infection - and to conduct a qualitative comparison of each CEP element across 6 CEP in participating countries (Germany, France, Ireland, the Netherlands, Sweden, and Scotland). With respect to this latter objective, we did not rate CEP overall, but rather identified similarities and differences between CEP by ranking of individual elements, and highlighted challenges encountered when comparing CEP from different countries or territories.

\section{MATERIALS AND METHODS}

\section{Definitions}

"Context" concerns the circumstances in a territory independent of the testing protocol that can influence the confidence in freedom from infection in a given animal, herd, or territory. Three main elements are relevant: information about the background BVDV situation (herd-level prevalence), the CEP, and cattle demographics. Information on the BVDV background situation and CEP information is based on the epidemiologically relevant population. For BVDV, this includes all dairy and beef herds where calves are born. We excluded other cattle types because they are often housed and thus pose a limited risk for transmission of the virus (e.g., veal and beef fattening cattle) or because the risk of transmission is considered very low compared with that of dairy and beef breeding herds (e.g., fattening of dairy cattle before slaughter). All 
CEP in our study solely focus on dairy and beef breeding herds. By decreasing the number of persistently infected animals $(\mathbf{P I})$ in breeding herds, the potential for PI to move into nonbreeding herds also decreases. However, we do account for the risk from other cattle types by including these herds in the information on cattle demographics (e.g., number of cattle herds and cattle density).

"Initial enrollment" describes the actions undertaken by a herd keeper from the time of enrollment of their herd into the CEP through to the time when BVDVfree status is obtained. This includes initial screening of the herd for presence of BVDV and any additional screening measures applied in the event of a positive test result or to prevent introduction of the virus.

"Surveillance" relates to those aspects of the CEP once BVDV-free herd status has been achieved and the herd is monitoring free status. This includes the definition of freedom, the test protocol for monitoring free status, the testing required to reestablish free status in the event of its being lost, and additional measures that minimize the risk of introduction of the virus through trade. This is based on the definition suggested by Hoinville et al. (2013), which was also adopted by The RISKSUR project (2015).

"Spot testing" tests for antibodies in a small representative group of young animals within the herd to indirectly indicate the presence of a PI in that management group and the animals within the herd with which they have contact.

"Bulk milk testing" tests bulk milk for antibodies to indirectly indicate the current or previous presence of a PI or for the presence of virus to directly indicate the presence of a PI.

"Ear notch testing" tests the skin of calves for virus within a few days after birth to detect PI. Sample collection is usually combined with the tagging of the calves.

\section{BVDV Control Programs}

The BVDV CEP are continually changing. This study is based on CEP in place in 2017, and subsequent changes (including, for example, the change to a compulsory BVDV CEP in the Netherlands at the beginning of 2018) are not included. A graphical representation of each specific CEP can be found in the Supplemental Files S1 to S6 (https://doi.org/10.3168/ jds.2019-16915). A more general description is included below.

Germany. In 1998, a voluntary BVDV CEP began, for which the individual Federal States were responsible. In 2011, a nationwide mandatory animal-level BVDV CEP based on tissue tag testing of calves was set in place (Wernike et al., 2017). The aim of this CEP is to detect and reduce the number of PI (Supplemental File S1; https://doi.org/10.3168/jds.2019-16915). In 2016, adjustments to the regulation were made to reflect experiences from the CEP to further reduce risk of transmission via trade, including a quarantine after the detection of a new case and trade restrictions for pregnant cows. Vaccination against BVDV is applied on a voluntary basis.

France (Brittany). No national standards for BVDV control in France exist, and each region can decide whether it wants to control BVDV and how to do it. In our comparison of CEP, we included Brittany, a region in the west of France where surveillance and control programs for BVDV have been implemented (Joly et al., 2005). Both programs are coordinated by Groupements de Défense Sanitaire (GDS), the regional animal health service. The surveillance program, in place since 2008, is mandatory. It is required for all cattle farmers to know their BVDV herd status by performing bulk milk testing in dairy herds or serological tests in beef herds. Since 2017, a voluntary CEP has been established for farmers who wish to eradicate BVDV from their herd as follow-up to the mandatory surveillance program. The aim of this CEP is to detect and eliminate PI in herds (Supplemental File S2; https://doi.org/10.3168/jds.2019-16915). Vaccination against BVDV is applied on a voluntary basis.

Ireland. A BVDV CEP based on tissue tag testing of newborn calves started in 2012 (Graham et al., 2014). Participation in the animal-level CEP was initially voluntary, but became compulsory on January 1, 2013. The CEP is industry-led and coordinated by Animal Health Ireland (AHI). Its target is to eradicate BVDV from Ireland before the end of 2020 (Supplemental File S3; https://doi.org/10.3168/jds.2019-16915). Vaccination against BVDV is applied on a voluntary basis.

The Netherlands. A voluntary industry-led BVDV CEP at the herd level based on bulk milk (in dairy herds) and individual blood testing for BVDV was in place between 1998 and 2018 (Mars and van Maanen, 2005; van Duijn et al., 2019). The aim of the CEP was to eliminate BVDV from herds by detecting and removing PI and monitoring the subsequent free status (Supplemental File S4; https://doi.org/10.3168/jds .2019-16915). Vaccination against BVDV is applied on a voluntary basis.

Sweden. Sweden is the only country in this study that has already achieved freedom from BVDV. In September 1993, a CEP was launched that aimed to eradicate BVD without vaccination. This is in contrast to the other territories included in this study, where vaccination is allowed. In 2001, a mandatory CEP required all cattle herds to be tested for BVDV on a 
regular basis (Hult and Lindberg, 2005). In 2008, few herds remained under investigation for BVDV, and risk-based surveillance was introduced. In 2011, the last case was detected, and by 2014, test results from the CEP indicated that Sweden was free from infection. This was confirmed in 2016 through a quantitative evaluation of surveillance results from 2012 to 2015 performed by SVA. The current surveillance program, based on antibody testing and surveillance at slaughter, started in 2017. This program is designed to detect the presence of infection at a herd design prevalence of $0.2 \%$, with $99 \%$ confidence (National Veterinary Institute, 2015; Supplemental File S5; https://doi.org/10 .3168/jds.2019-16915).

Scotland. Scotland is 1 of 4 countries in the United Kingdom; each country has its own compulsory or voluntary CEP. Our study focuses on the BVDV CEP in Scotland. The industry-led BVDV CEP in Scotland is mandatory and based on spot testing. The CEP has had 4 stages to date: (1) subsidized screening of the herd for BVDV from September 2010 to April 2011; (2) mandatory screening of all breeding herds by spot testing for antibodies or antigen testing of calves, with all breeding herds to be screened by February 1, 2013, and annually thereafter; (3) control measures (e.g., movement restrictions) that came into force in January 2014; and (4) enhanced testing and further movement restrictions that were were implemented on June 1, 2015 (Scottish Government, 2016). The aim of the CEP is to eradicate BVDV from Scotland (Supplemental File S6; https:/ /doi.org/10.3168/jds.2019-16915). Vaccination against BVDV is applied on a voluntary basis.

\section{Data Collection}

An existing tool, RISKSUR (The RISKSUR Project, 2015; Comin et al., 2016) was used to ensure harmonized data collection from each participating country or region (Germany, France, Ireland, the Netherlands, Sweden, and Scotland), hereafter referred to as territories, about both the target hazard BVDV and the CEP. RISKSUR is a digital tool built to support the design and evaluation of surveillance systems. The tool guides the user through all steps that should be considered when designing a surveillance system, including the surveillance objective, target population, surveillance enhancements, testing protocol, study design, sampling strategy, data generation (sample collection), data/ sample transfer, data translation (sample analyses), epidemiological analyses, dissemination of results, and surveillance review (The RISKSUR Project, 2015; Comin et al., 2016).

RISKSUR is used as a tool for detailed descriptions of surveillance programs. Because we were interested in control and all country-specific aspects that are relevant to assessing confidence in freedom, the RISKSUR tool was expanded for the current study to also collect information on aspects of control and context, such as actions taken following positive test results and risk factor occurrence. The expanded RISKSUR tool (RISKSURexp) included risk characteristics, structure of the cattle industry (i.e., size, production system, trade), CEP history and development, organizations involved, biosecurity measures, and results of the BVDV CEP. To gain a comprehensive overview of the situation in each territory, the tool was completed in early 2018 by consortium members of STOC free, supported by animal health authorities for each of the territories covered in the STOC free project (van Roon et al., 2019; https: //www.stocfree.eu/); data provided show the contexts and BVDV CEP in place in 2017.

All information was grouped under 3 main topics: (1) context (i.e., BVDV status, structure of the cattle industry, occurrence of risk factors); (2) initial enrollment (actions required to obtain a BVDV-free herd status); and (3) surveillance (measures applied to monitor herdlevel BVDV-free status).

\section{Data Analysis: Comparative Ranking}

Separate data files were created for each CEP, containing qualitative information about all aspects of CEP, risk factor occurrence, and context. All 6 data files were compared to identify differences and similarities. For each topic (context, initial enrollment, and surveillance), a list was created of elements that could influence the confidence of freedom from BVDV in the herd (Supplemental File S7; https://doi.org/10.3168/ jds.2019-16915). Then, beginning with context, each element was considered in turn, and, where relevant (as described below), the territories were ranked relative to each other using scales from 1 (most optimal situation) to 6 (least optimal situation) based on a trend consistent with increasing difficulty to achieve herdlevel confidence of freedom. All elements were ranked separately and independently of the other elements. When the value of an element was similar between territories, the same rank was assigned to these territories and the ranking was condensed (e.g., ranked only from 1 to 3). Thus, a rank of 1 represented the territory with the most optimal situation for that particular element [e.g., the lowest risk of introduction or transmission of BVDV into the herd (context) or the highest probability of detection (outcomes of initial enrollment and surveillance)], each being important contributors to herd-level confidence of freedom. Sweden was not included in the ranking of elements relating to the third topic (surveillance), given that it is expected to 
be BVDV-free, and its surveillance approaches are considerably different from those of territories currently working toward freedom. Some assessed elements were excluded from the comparisons or ranking: (1) elements presenting valuable information about the context or the CEP but without direct influence on confidence of freedom, such as the program level; (2) elements with (almost) no variation between territories, such as the proportion of cattle herds that graze; and (3) elements for which few or none of the few territories possessed reliable information, such as the number of professional visitors on a farm, even though these were indicated as risk factor in several territories.

\section{RESULTS}

All information relevant to comparison of the 6 BVDV CEP and their subsequent rankings are presented in Tables 1, 2, and 3. The context elements, including the background BVDV situation, the CEP, and cattle demographics, are presented and ranked in Table 1. The initial enrollment elements in the 6 CEP, including initial screening of the herd for presence of BVDV and any additional screening measures applied in the event of a positive test result or to prevent introduction of the virus, are presented and ranked in Table 2. Territories where all herds enrolled in the CEP in previous years (all relevant herds are already participating) were excluded from Table 2. This, for example, is the case for Sweden (which has already achieved freedom from BVDV) and for Germany, Ireland, and Scotland (which began their compulsory CEP before 2017). The surveillance elements are presented and ranked in Table 3, including the definition of freedom, the test protocol for monitoring free status, the testing required to reestablish free status in the event of its being lost, and additional measures that minimize the risk of introduction of the virus by trade. The territory expected to be free of BVDV (Sweden) is not included in the ranking because its surveillance cannot be ranked relative to the surveillance of territories currently working toward freedom-Germany, France (Brittany), Ireland, the Netherlands, and Scotland-because their surveillance is designed for a different purpose. In Supplemental File S7 (https://doi.org/10.3168/jds.2019-16915), the rationale behind the ranking is explained for each element presented in Tables 1-3.

\section{Context: BVD Situation}

Herd-Level Prevalence of BVDV in Breeding Herds. In 2017, the territories involved in this study differed greatly in their BVDV herd-level prevalence: the higher the herd-level prevalence, the greater the risk of introduction of the virus into a susceptible herd. This ranged from zero in Sweden to $10.4 \%$ in the Netherlands (Table 1). Sweden was ranked best [1] because it had the lowest risk of transmission of BVDV between herds.

Application of $B V D V$ Vaccination. In all territories except Sweden, vaccination against BVDV is applied on a voluntary basis (Table 1). As vaccination can affect test results (e.g., on antibody testing in bulk milk), territories in which such testing schemes are applied take this into account in their CEP. In the Netherlands, it is not possible to screen bulk milk for virus by PCR until at least $23 \mathrm{~d}$ after live-virus vaccination, as the PCR test may detect vaccinal virus and generate a false-positive result. Additionally, unvaccinated animals must be selected for serological screening and a farm should only start vaccination after removal of all PI. Thereafter, when monitoring the BVDV-free status, screening for BVD antibodies (spot test) can be performed after vaccination of the herd, provided that the youngstock selected for the spot test have not been vaccinated. In Scotland, there are guidelines with regard to the animals that the farmer can select for testing in vaccinating herds. Ideally, unvaccinated animals should be tested but if all appropriate animals are vaccinated, then information about the date of vaccination and type of vaccine must be provided alongside the sample to facilitate interpretation of the results of the test. In Ireland and Germany, vaccination does not have consequences for the testing schemes because all newborn calves are tested for virus; in Brittany, this is also taken care of by an alternative PI detection program. Within the context of becoming free from infection, the production of false positives (i.e., infection free but seropositive because they are vaccinated) is not directly relevant, because the focus is on false negatives. However, false-positive results could lead to a waste of resources.

\section{Context: Program Information}

Program $\operatorname{Aim}(s)$. The CEP in the different territories were designed to achieve different program goals. For instance Sweden, now BVDV-free, has a CEP in place to detect BVDV after re-introduction. The CEP in Germany, Ireland, and Scotland aim to eradicate BVDV from the territory. The voluntary CEP in the Netherlands and Brittany aim to eradicate BVDV at the herd level (Table 1).

Program Level. Control and eradication programs that test at the animal level can be distinguished from those that test at the herd level (Table 1). Germany 
van Roon et al.: BVDV CONTROL PROGRAM ELEMENTS INFLUENCING CONFIDENCE OF FREEDOM

4659

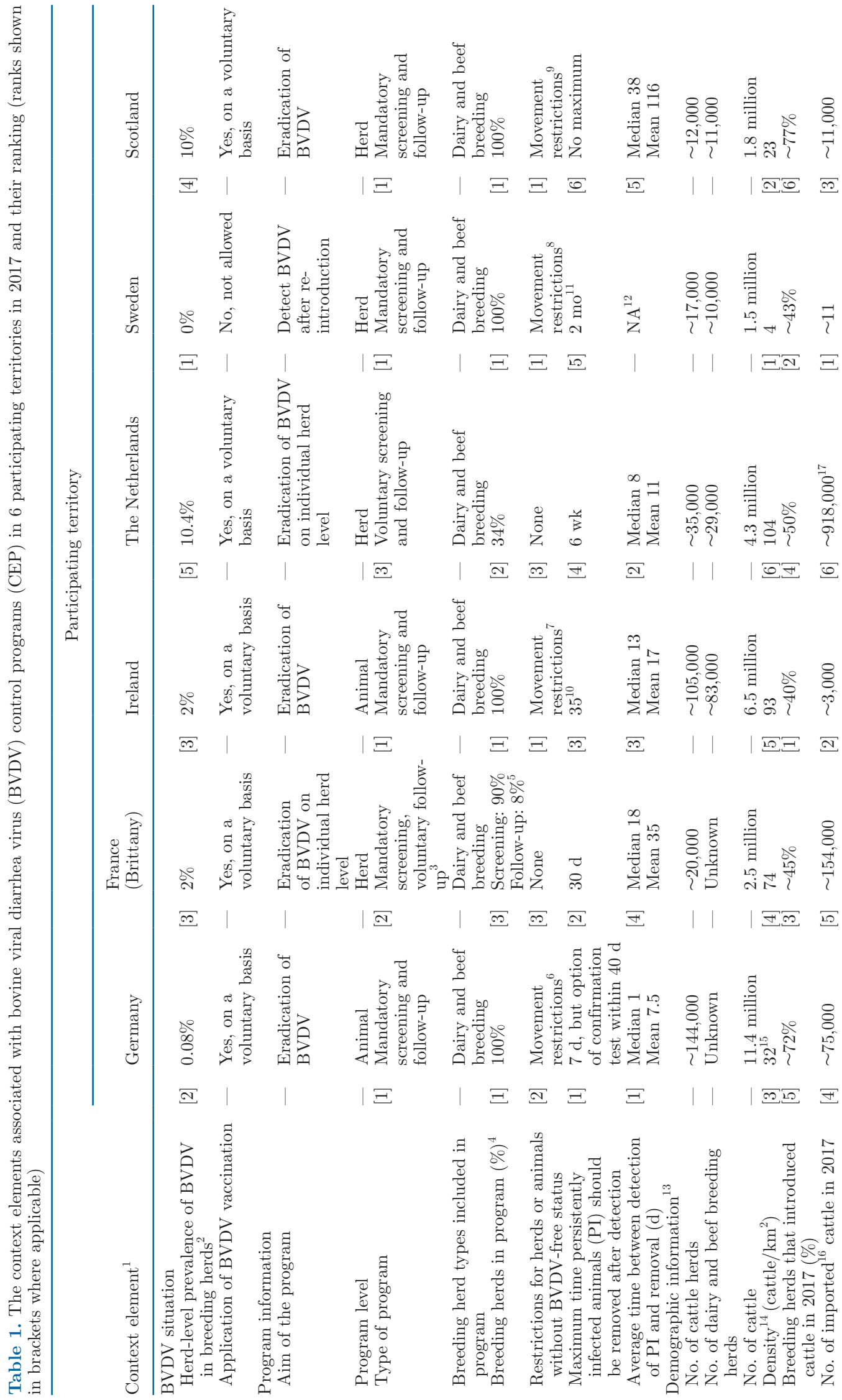


van Roon et al.: BVDV CONTROL PROGRAM ELEMENTS INFLUENCING CONFIDENCE OF FREEDOM

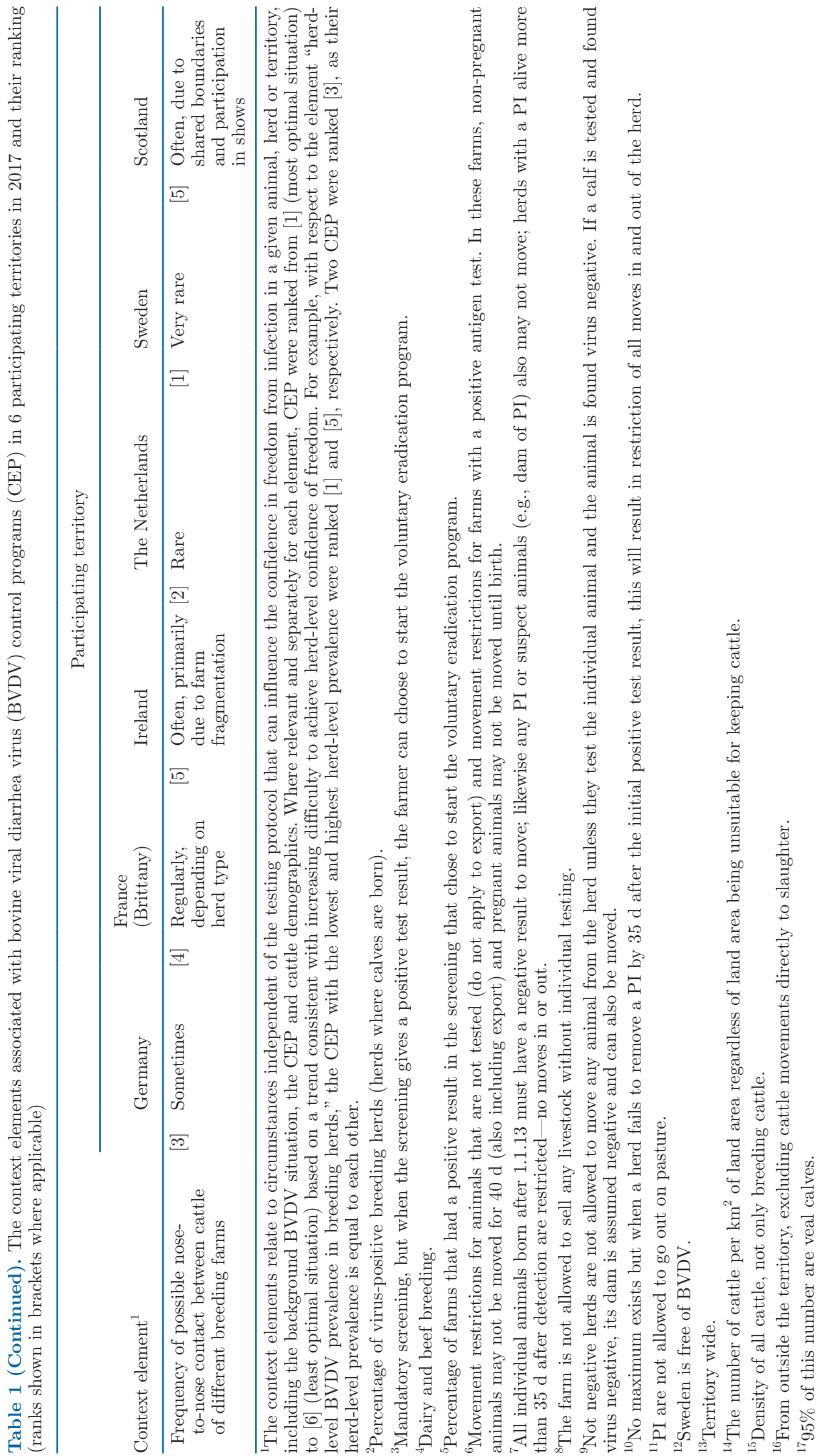




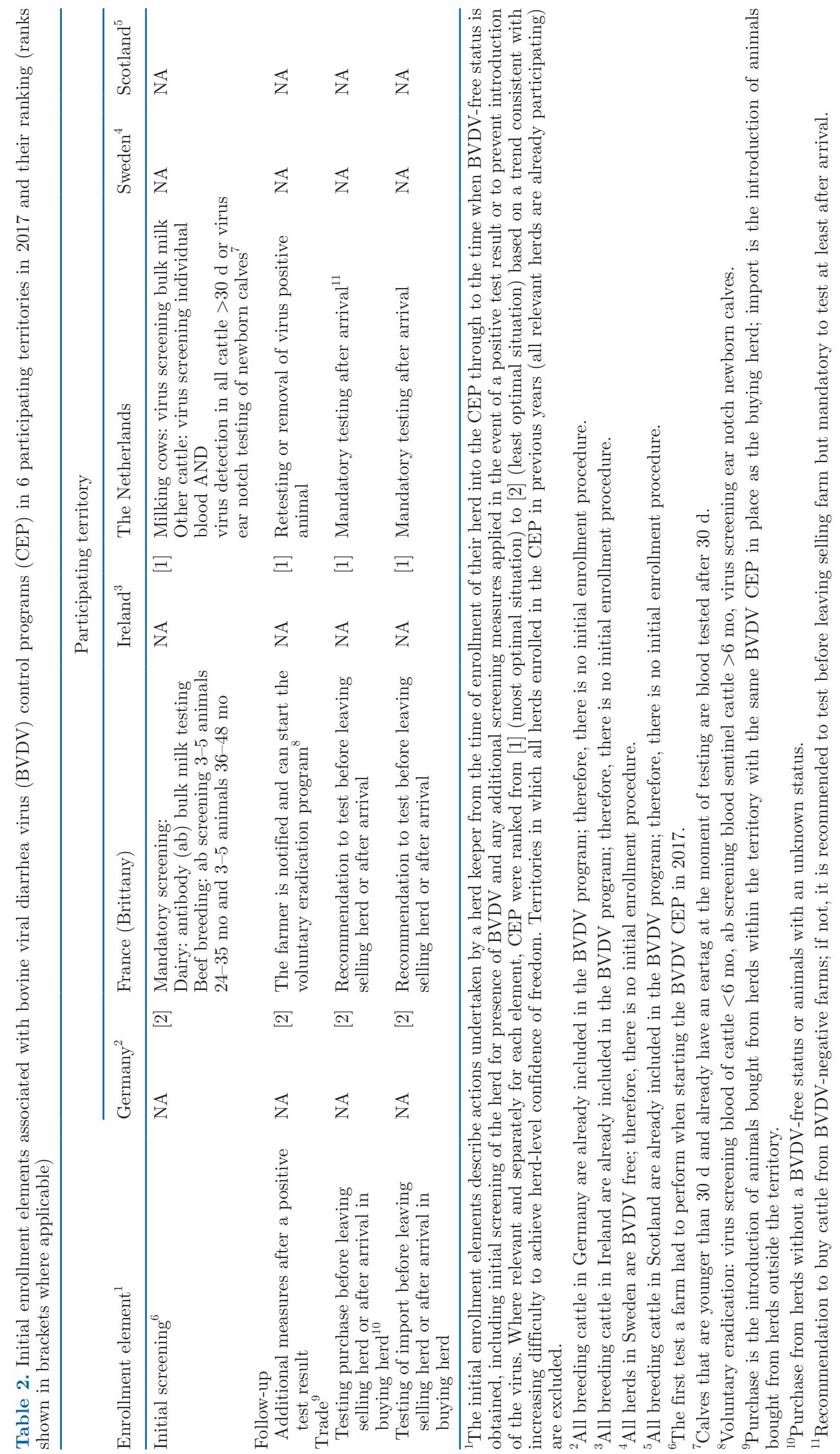


van Roon et al.: BVDV CONTROL PROGRAM ELEMENTS INFLUENCING CONFIDENCE OF FREEDOM

4662

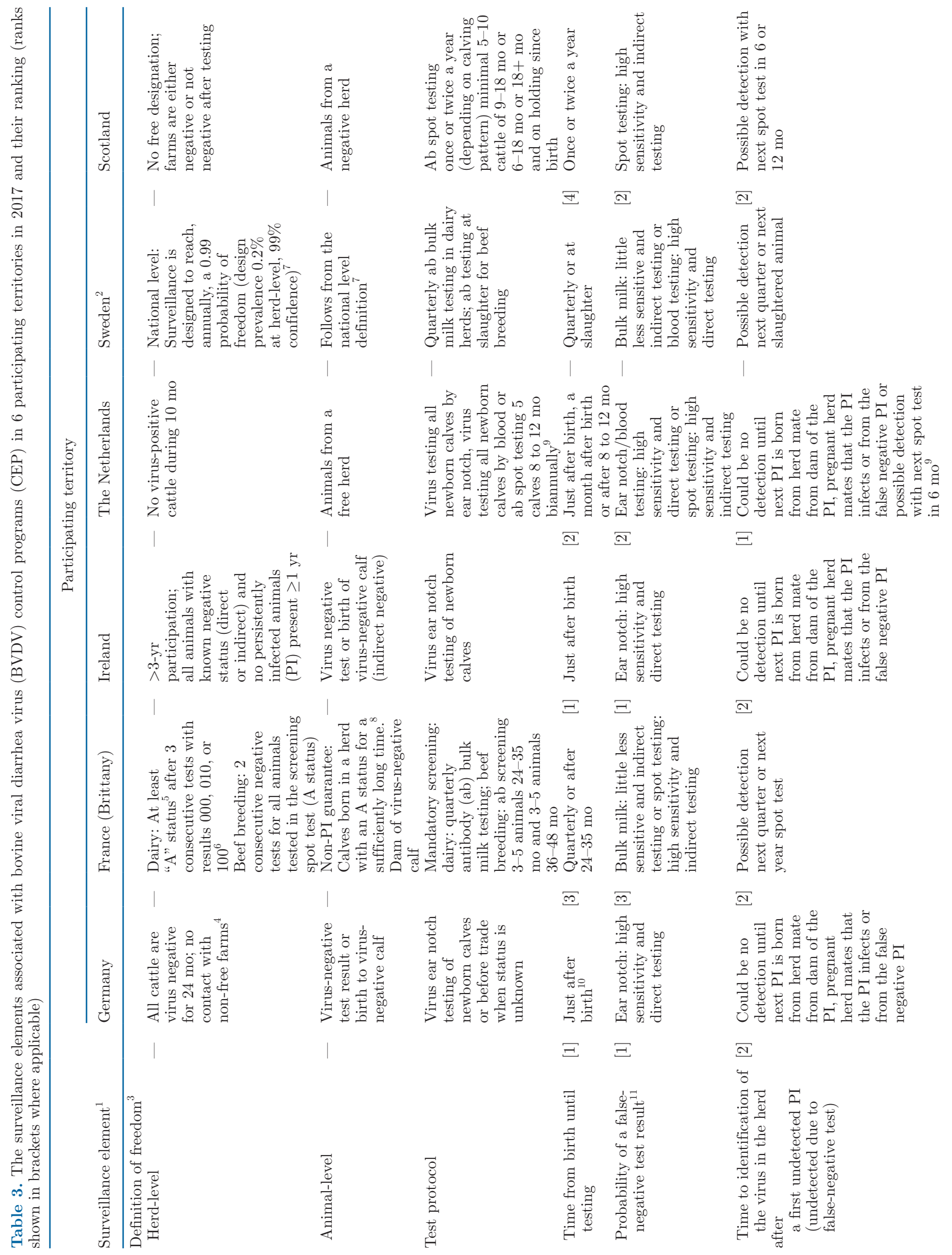


van Roon et al.: BVDV CONTROL PROGRAM ELEMENTS INFLUENCING CONFIDENCE OF FREEDOM

4663

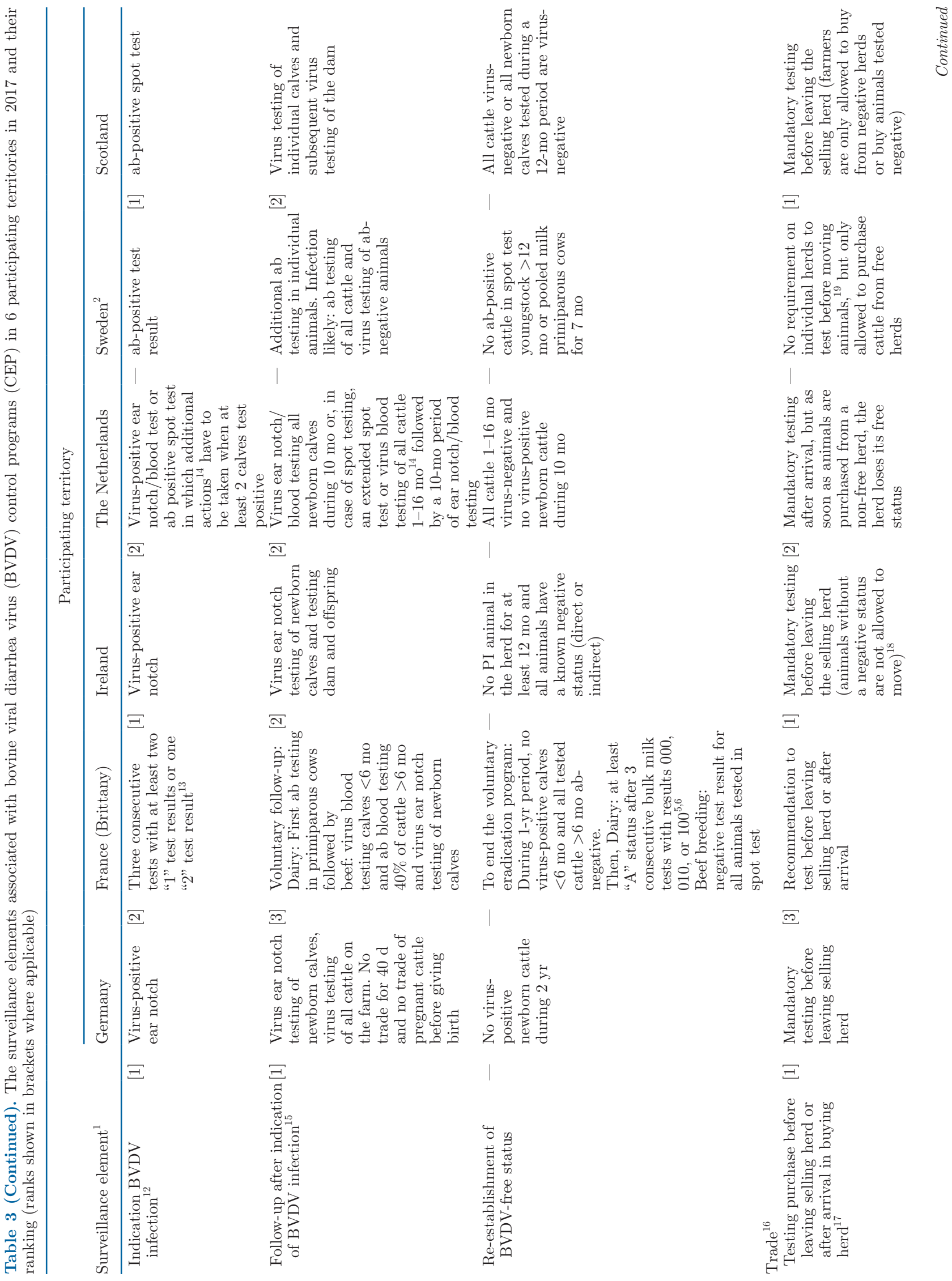




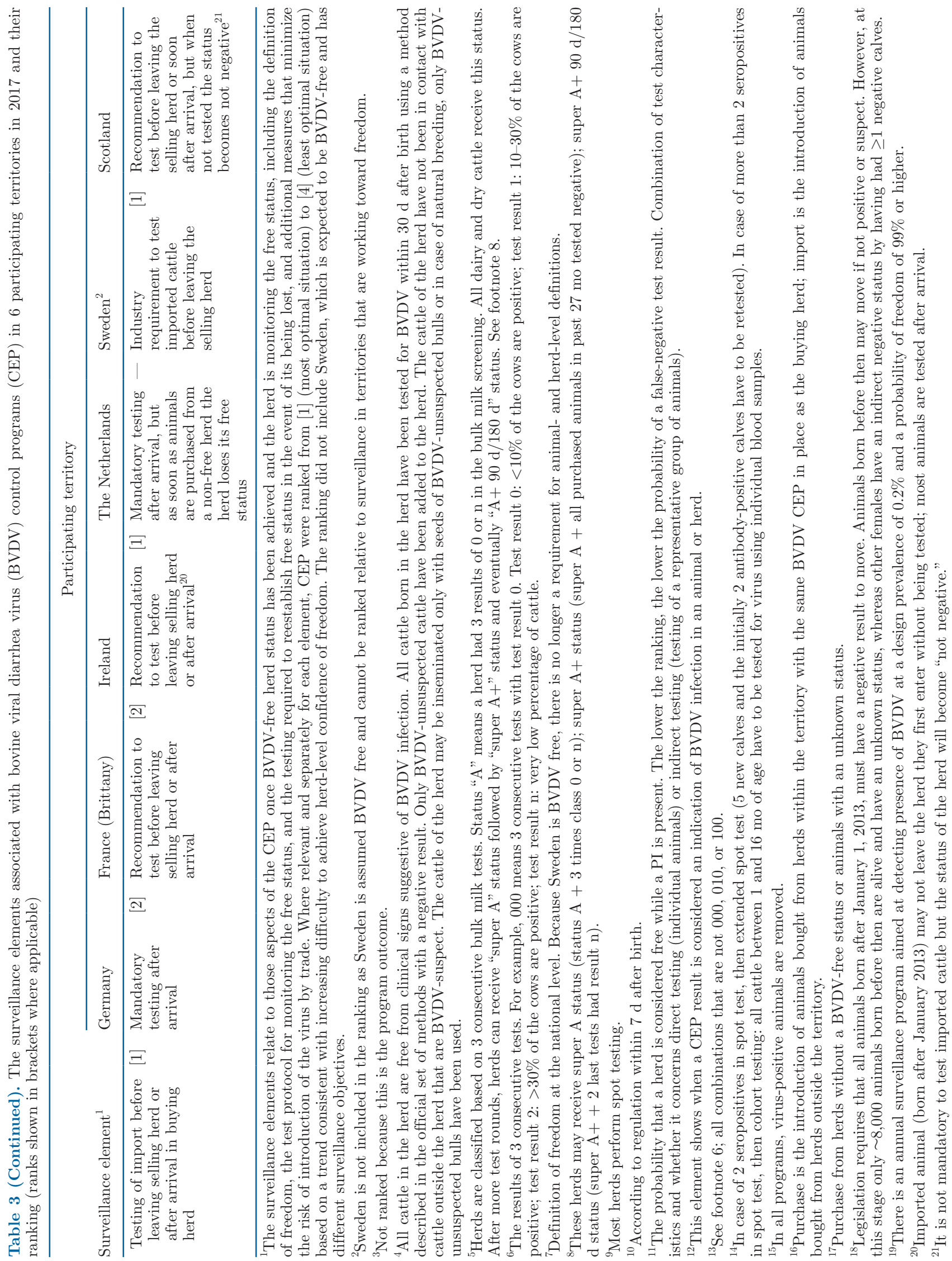


and Ireland test individual animals and assign free status to individual animals that test negative for BVDV. The other territories perform a testing protocol at the herd level and assign free status to the herd. However, although a CEP is designed at the animal or herd level, within a herd-level CEP, free status may also be assigned to individual animals and vice versa. For example, Ireland assigns free status to both herds and individual animals, and herd-level programs may assign free status to individual animals. Because it was impossible to conclude which of these program levels (either herd or animal) is optimal, this element was not ranked.

Mandatory or Voluntary. The Netherlands had a voluntary CEP in 2017, whereas mandatory CEP were introduced in Sweden (2001), Germany (2011), Ireland (2013), and Scotland (2013). In Brittany, cattle farms are required to know their BVDV status, but although there is a mandatory surveillance CEP, they can choose to eradicate BVDV from their farm with the voluntary eradication program. Mandatory CEP have a better ranking than voluntary $\mathrm{CEP}$, because all herds in the epidemiologically relevant population are obliged to participate in the CEP and carry out control measures for BVDV (Table 1).

Herd Coverage. Control and eradication programs are developed to cover the epidemiologically relevant population. For BVDV, PI calves are the key to transmission, so the population of interest is all herds in which calves are born. All CEP include both dairy and beef breeding herds; however, the percentage of dairy and beef breeding herds included in each program varies. Mandatory CEP cover $100 \%$ of the relevant population whereas coverage in voluntary CEP is lower. In the Netherlands, $34 \%$ of breeding herds, mainly dairy herds, are covered, whereas in Brittany, only $8 \%$ of the farms that had a positive result in the bulk milk screening started the voluntary eradication program. Herd coverage is ranked worse in the territories with lower coverage (Table 1).

Herd Restrictions. All territories with a mandatory CEP have movement restrictions in place for herds or animals that do not yet have free status. All mandatory CEP prohibit movement of animals that do not have an individual negative test result when originating from a farm without free status (herd restrictions are specified in Table 1). The voluntary CEP only have movement restrictions for herds that participate in the CEP. Territories with movement restrictions are ranked better than territories without such restrictions because these restrictions lower the probability of transmission of BVDV from a possibly infected herd to a susceptible herd. Germany was ranked worse than other territories for movement restrictions because its movement restrictions for untested animals do not apply to export. However, the movement restrictions Germany has in place for farms with a positive antigen test do apply to export.

Removal of PI. Some CEP prescribe a maximum time from PI detection to removal, ranging from $7 \mathrm{~d}$ to 2 mo. Increasing the number of days that a PI stays on the farm increases the risk of transmission. Reducing the maximum time improves the ranking of the CEP. The actual time in days between detection and removal of a PI, which had a different ranking than the prescribed maximum time between detection and removal, was also included. The median number of days ranged from 1 to 38 (Table 1 ).

\section{Context: Demographic Information}

Cattle Population. The total number of cattle herds ranges from approximately 12,000 in Scotland to 144,000 in Germany (Table 1). When only looking at breeding herds, it ranges from approximately 10,000 in Sweden to 83,000 in Ireland. In Germany and Brittany, no distinction could be made between breeding herds and other cattle herds. The number of cattle ranges from approximately 1.5 million in Sweden to 11.4 million in Germany. This information was not ranked but the more relevant element "cattle density" was. Territories with a low cattle density were ranked better than countries with a high cattle density because the probability of spread of BVDV by contact between cattle is lower. Sweden ranked best with a cattle density of 4 cattle per $\mathrm{km}^{2}$ of land area and the Netherlands ranked worst with a cattle density of 104 cattle per $\mathrm{km}^{2}$ (Table $1)$.

Risk Factors for Transmission and Introduction of $B V D V$. A known risk factor for introduction of BVDV is introduction of cattle into the herd. We included the percentage of herds that introduced cattle in 2017 ("purchased" if from within the territory; "imported" if from outside the territory; Table 1). Ireland ranked best with $40 \%$ of the herds purchasing cattle on an annual basis, and Scotland ranked worst with $77 \%$. The number of cattle imported ranged from 11 in Sweden to 918,000 in the Netherlands. It should be noted that $95 \%$ of cattle imported into the Netherlands are veal calves, which are likely less relevant for transmission of BVDV, except for herds that keep veal calves and breeding cattle in the same location. Another known risk factor for transmission of BVDV between herds is direct contact between cattle from different herds. The possibility and frequency of nose-tonose contact between cattle of different breeding herds 
depends on the distance between pastures, the type of boundary, type of cattle, attendance at shows, and so on. Most territories do not have quantitative data available for this element; therefore, it was estimated by expert opinion (Table 1). Sweden ranked best because contact between cattle of different farms is very rare. Ireland and Scotland were ranked worst, primarily as a consequence of farm fragmentation and possibly extended grazing and attendance at cattle shows. It should be noted, however, that farmers that visit cattle shows are often pedigree breeders who may take greater care of biosecurity, thereby mitigating the risk, at least to some extent.

\section{Initial Enrollment}

Initial Screening. In Brittany and the Netherlands, the initial screening strategies are very different; for example, screening for antibodies versus virus, direct (individual) versus indirect testing (group), and different age groups and sample types tested (Table 2). The initial screening of the Dutch CEP was ranked best because all cattle are tested for virus, although a bulk milk sample is used to test lactating cows for virus in dairy herds. Brittany was ranked worst because not all cattle are directly screened.

Follow-Up. This element shows the measures taken when positive animals are detected in the initial screening (Table 2). In the Netherlands, for a herd to be allowed to continue with the CEP, PI should be removed. In Brittany, farmers have no obligation to remove PI. However, the farmer can also choose to start the voluntary eradication program, through which they also have to detect and remove all PI.

Trade. To minimize the risk of introducing BVD virus into herds, CEP in both Brittany and the Netherlands recommend or require herds to test introduced cattle (Table 2). However, their CEP differ as to whether this is recommended (Brittany) or mandatory (the Netherlands), and whether the introduced animal needs to be tested before leaving the selling herd or after arrival in the buying herd. The Dutch program ranked best because testing is mandatory. Neither program requires herds to test or quarantine their introduced animals before arrival in the herd (when herds are in the initial enrollment phase).

\section{Surveillance: Definition of Freedom}

The CEP vary in the way that infection-free status is defined - at the territory, herd, or animal level (Table 3 ). Sweden is the only territory that has a definition of freedom at the national level because BVDV is con- sidered absent so there is no longer a requirement for a herd-level definition of freedom. In Sweden, not all herds are necessarily tested annually, because surveillance is based on a combination of random and riskbased sampling, but all samples have to be antibody negative. In Germany and Ireland, when all animals in a herd have tested negative for BVDV and have an animal-level definition of freedom, this leads to a herdlevel definition of freedom. In Brittany, a herd-level free status is assigned, and animals within a free herd can obtain a non-PI guarantee [see Table 3, Supplemental File S7 (https://doi.org/10.3168/jds.2019-16915), and Joly et al., 2005, for detailed information]. In the Netherlands, a herd-level free status is assigned, and all animals within those herds are assumed BVDV free. In Scotland, farms are classified as either negative or not negative after testing; they do not use the designation "free status." The definition of freedom was not ranked because these are overall outcomes of each CEP and the result of detailed elements that have already been ranked.

\section{Test Protocol}

The test protocol in each of the territories after achieving a herd-level or animal-level free status is described in Table 3. The test protocol itself was not ranked because its success depends on many different factors. We instead ranked the probability that the test protocol would detect the virus. We also ranked the follow-up after indication of a BVDV infection and the route to re-establishment of free status.

Time From Birth to Testing. The first aspect of the test protocol that was ranked was the time between birth of a calf and the first test event (Table 3). If this calf is a PI, this time should be as short as possible, to prevent further transmission of the virus. Farmers who monitor their free status by ear notch testing will normally test their calves within a few days of birth. Herds that apply bulk milk testing or spot testing will detect a new PI later, depending on the frequency of testing and the promptness of further investigations following initial serological evidence of infection. The territory in which the time from birth to testing is shortest is ranked best.

Probability of a False-Negative Test Result. The second aspect of the test protocol that was ranked was the probability of a negative test result when a PI was present (Table 3). This probability depends on the sensitivity of the diagnostic test and whether it concerns direct testing (individual animals) or indirect testing (testing of a representative group of animals). Ear notch testing was ranked better than either anti- 
body bulk milk or spot testing, because it is individual testing. Antibody bulk milk testing was ranked worse than ear notch testing and spot testing, because its sensitivity is reduced by both the dilution of positive samples and by animals that could be missing from the bulk sample.

Time to Identification of Virus in the Herd After a First Undetected PI. The third aspect of the test protocol that was ranked was the time until the virus was detected in the herd after the first PI was missed because of a false-negative test result (Table 3). Here, we ranked the spot test (performed at least twice a year) better than the ear notch test. Given that the efficiency of virus transmission by a PI is very high, the presence of a PI usually results in widespread seroconversion in herd mates. Depending on the distance between PI and susceptible herd mates (Houe et al., 2006), we assume that the virus will be detected by the next spot test. With the ear notch test, either a next PI calf needs to be born or susceptible pregnant cattle have to become infected and give birth to a PI calf, which on average could result in slightly later identification of the virus than biannual spot testing. Therefore, the Netherlands was ranked best based on the assumption that the antibody prevalence reaches $50 \%$ within a short time $(<1 \mathrm{mo})$, followed by the other territories with ear notch testing (Germany and Ireland), less frequent spot testing (Scotland), or quarterly bulk milk testing combined with less frequent spot testing (Brittany).

\section{Indication of BVDV Infection}

This element shows when a CEP result is considered an indication of BVDV infection in an animal or herd (Table 3). Every virus-positive test result (in Germany and Ireland) or every antibody-positive test result (in Sweden and Scotland) is assumed to be a BVDV infection. In Brittany, free status is assigned after 3 consecutive bulk milk tests in which one of the tests is allowed to be antibody positive (details in Table 3). In the Netherlands, farmers either perform a spot test in which 5 animals are tested or they test newborn calves by blood or ear notch. In herds that choose to perform the spot test, additional actions have to be taken when at least 2 animals test seropositive (details in Table 3). Therefore, Brittany and the Netherlands are ranked worst.

Follow-Up After Indication of BVDV Infection. In all territories, PI have to be removed before BVD free status can be regained. Most territories, after removing the PI, follow their initial test protocol. The territories that have additional measures in place, such as testing the dam of the PI, are ranked better. In Brittany, farms can choose to participate in the voluntary eradication program after losing their free status following the detection of BVD antibodies in bulk milk. If the farm does not want to eradicate BVDV, it continues performing bulk milk testing (Table 3 ).

Re-Establishment of BVDV-Free Status: Definition of Freedom. This element shows the protocol for re-establishing herd-level free status after removing the PI and performing additional measures if included in the CEP (Table 3). The territories differ in test protocol and in the duration of the period in which no antibody- or virus-positive animals should be found to re-establish free status; this ranges from 7 mo to $2 \mathrm{yr}$. As this duration depends on previous measures and the context, this element was not ranked.

\section{Surveillance: Trade}

Trade is a known risk factor for introduction of BVDV into a farm or territory. As in the initial enrollment phase, all CEP recommend or require free herds to know or test the BVDV status of introduced cattle (Table 3). Except in Brittany, where it is only recommended, it is mandatory to test cattle purchased from non-BVDV-free herds within the territory. In Germany, Ireland, and Scotland, which are ranked best, cattle should be tested before they leave the selling herd, because animals without a negative status are not allowed to move or farmers are only allowed to buy cattle from herds with BVDV-free status. In the Netherlands, it is mandatory to test purchased cattle, although this can be conducted following their arrival on the farm. In Sweden, no requirement exists to test purchased animals on individual herds, but only cattle from free herds can be purchased. Control and eradication programs do not describe measures such as quarantine to reduce the risk of introducing a pregnant cow carrying a PI or a transiently infected cow. For imported animals, territories with mandatory testing after arrival are ranked best, because none of the CEP require imported animals to be tested before their arrival on the farm. In Sweden, it is an industry requirement that imported cattle be tested before arrival.

\section{DISCUSSION}

In this study, we present a detailed overview of those elements of CEP that are relevant to the assessment of confidence in freedom. In this work, we used BVDV as a case study, noting that many countries or regions in the world have implemented their own CEP. We considered BVDV CEP in 6 different territories within 
Europe to capture differences and similarities and to describe and compare the elements of CEP that contribute to confidence of freedom (the likelihood that a bovine from a herd categorized as BVDV-free is truly free from infection).

Many factors influence confidence of freedom. In this study, we considered all factors that differed between CEP, including context elements, because they appeared to be essential in the comparison of CEP. Many elements are interrelated; therefore, it was not possible to determine the relative contribution of each element to the overall confidence of freedom. Therefore, CEP comparisons were restricted to individual elements, and no aggregation was attempted. The CEP can be compared in different ways. They are usually compared by focusing on the current status and epidemiological or economic features of the disease (Greiser-Wilke et al., 2003; Moennig et al., 2005; Houe et al., 2006), but CEP have also been reviewed in terms of the financial and economic implications of prevention and control measures (Pinior et al., 2017). Instead of primarily focusing on comparing programs, studies of CEP outline key aspects of control activities (Houe et al., 2006; Geraghty et al., 2014). We felt that a more detailed comparison of BVDV CEP was needed, and have focused, for the first time, on differences between elements within CEP that could influence the confidence in freedom from BVDV infection in the herd.

\section{Context}

We identified substantial differences in BVDV CEP. These differences partially reflect differences in context, such that each CEP is tailored to the specific situation in a country (Sandvik, 2004; Moennig et al., 2005). Reasons for these differences can also relate to other factors, such as political realities, cost efficiency, human behavior, or cultural differences (Lindberg and Houe, 2005; Heffernan et al., 2009). This strongly suggests, in agreement with earlier studies (More et al., 2009; Schuppers et al., 2012; Toftaker et al., 2018), that context-specific key factors influence the risks of introduction and must be taken in account in any analysis meant to develop a method to compare the probability of freedom offered by different CEP.

Our approach to ranking different CEP elements should thus be interpreted with caution, because different contexts could easily change such a ranking. For example, the comparison of cattle densities in this study was based on the number of cattle per $\mathrm{km}^{2}$ of land area, regardless of land area being unsuitable for keeping cattle. In some territories, such as the Netherlands, almost all land area is suitable for keeping cattle, and cattle herds are fairly evenly distributed throughout the country. However, in other territories, such as Sweden, Scotland, and Ireland, the spatial distribution of cattle herds is heterogeneous. The ranking could therefore be different when distinguishing between low- and highdensity areas within the same territory.

\section{Complexity of Ranking}

It could be argued that some elements should not be ranked at all in this study because they are influenced by too many factors. One example is the surveillance element "Probability of a false-negative test result (while there was a PI present)." The probability of a falsenegative test result can also be influenced by factors within the laboratory; for example, by human error, testing protocol applied (pooled sample or not, PCR or ELISA), or the presence of maternally derived antibodies (Fux and Wolf, 2012). In addition, the probability of a false-negative test result can be influenced by factors that operate before the point of laboratory testing. With ear notch testing, these could be factors such as interval from collection to submission of the sample, time spent in the postal system, or deliberate interference by the farmer. For spot testing, this could relate to nonrepresentative cohort sampling or neglecting to sample all separately managed groups of the target age, among others. Relevant to trade, animals from a birth cohort could be sold before spot testing is carried out, which is often the case with dairy bull calves.

Another element that was very challenging to rank was "Time until identification of the virus in a herd where the first PI was undetected due to a false negative test result." We decided to rank biannual spot testing as better than ear notch testing because the time until virus circulation is detected after the initial false-negative result may be shorter on average than that with ear notch testing. Further, spot testing is able to identify virus circulation when the PI itself is already removed from the herd (death or moved off-farm to a fattening unit). Whether a spot test is timelier than ear notch testing, however, depends on many factors, including the frequency of spot tests in the young animal group. In the case of biannual spot testing, it is assumed that spot testing will detect virus circulation faster; however, in some countries, spot testing is performed only once a year. In these cases, ear notch testing may result in earlier detection of virus circulation in the herd. Another factor will be farm management. For example, if age groups have no direct contact, the probability of detecting antibodies with the next spot test is much lower. Additionally, in a herd with concentrated calving (e.g., spring calving), the minimum time 
between the primary case (birth of a PI but undetected due to a false-negative test) and secondary case(s) (birth of additional PI as a consequence of the primary case) would be approximately $12 \mathrm{mo}$. In a year-round calving herd, the minimum time from primary to secondary cases is likely to be shorter. A third factor is the design prevalence chosen to determine the number of animals to be selected for testing. The period for detection of infection using the spot test will be prolonged by the time until the design prevalence is reached. If a design prevalence of $50 \%$ is chosen, the time until detection of virus circulation in the spot test will depend on both the testing frequency and the time that it takes to reach the design prevalence of $50 \%$ in the target group (youngstock). It is well known that a PI is highly infectious and effectively transmits the virus to all other cattle in the cohort within a very short period. Nevertheless, if different age groups within a herd are housed separately, it may take time for the virus to spread between age groups. In such cases it could take more than 1 yr until the virus is transmitted throughout the cattle herd and design prevalence is reached. The time until identification of the virus in the herd is reduced with both ear notch and spot testing when multiple PI are born in the same birth cohort (quick detection of the next PI). When only a single PI is born and tests false negative with ear notch testing, the virus may be detected after 6 to 8 mo if the PI infects other susceptible pregnant cows or after at least 24 mo when the PI itself calves. This shows the difficulty of ranking this element and highlights the detailed data needed to be able to make a valid comparison.

In our study, we applied an approach in which we compared the same elements between different CEP. The ranking process led to very valuable discussions between partners in the STOC free project because each partner was provoked to think carefully about each element within their CEP relative to other CEP. The intensive and comprehensive discussions provided insight in the reasoning behind the design of different CEP in different countries and added to the scientific level of the discussion.

\section{Challenges for Comparison}

The RISKSURexp tool allowed us to collect very detailed information about BVDV control and context in the 6 territories included in this study (The RISKSUR Project, 2015; Comin et al., 2016). This tool proved very valuable as a means to precisely define the data of interest and collect information in such a way that allowed comparison between territories. Collecting information to allow direct comparison was indicated as a challenge in a review of Johne's disease control activities in 6 countries (Geraghty et al., 2014). In our study, we found that in some territories all data were readily available, whereas in others, access to the data was difficult or the required data were not collected. Especially challenging for data collection were differences in the way that territories recorded data. For example, for a seemingly easy to collect element such as "the number of dairy and beef herds," it was very difficult to obtain comparable data from different territories. Some territories categorized every farm where milk was delivered as a dairy herd, even though beef cattle were also present, whereas other territories made a clear distinction between dairy, beef, and mixed herds or even other herd categories. When methods are developed to determine the confidence in freedom from infection resulting from CEP, these differences between data will need to be addressed. The uncertainty around the confidence in freedom resulting from CEP might be affected by the ease with which data can be accessed on the herds participating in the CEP.

Another challenge for comparison was that the territories included in this study were at very different phases of control or eradication. Territories with programs that have been in place longer have gone through several stages of control with varying aims and strategies. For example, Ireland (Graham et al., 2014) and Scotland (Scottish government, 2016) each commenced with voluntary screening that subsequently evolved into mandatory CEP. As these programs progress toward eradication, additional control measures are coming into force. The suitability of a test strategy in a certain stage of control, and thus the resulting confidence of freedom, is highly dependent on the specific aim addressed at that time (Houe et al., 2006). This is also the reason for not ranking Sweden. Because Sweden is free from BVDV, a less strict CEP is sufficient because the only risk of introduction is through external introduction. However, if BVDV were to be imported into Sweden (e.g., an animal tested false negative), the consequences could be substantial. This highlights the difficulties involved in comparing CEP.

\section{CONCLUSIONS}

We identified considerable heterogeneity in the elements of CEP that influence confidence of freedom, with respect to both the context and individual control strategies, among the $6 \mathrm{CEP}$ that were evaluated. In this study, both description and ranking were used, with ranking allowing us to highlight heterogeneity in a manner that is clearer than using description alone. The similarities and differences in context, initial en- 
rollment, and surveillance strategies in the different territories that we have identified here will need to be incorporated into a common framework aimed at quantitative comparison of confidence of freedom from infection.

\section{ACKNOWLEDGMENTS}

This work was carried out with the financial support of the Dutch Ministry of Agriculture, Nature and Food Quality and is part of the STOC free project that was awarded a grant by the European Food Safety Authority (EFSA) and was co-financed by public organizations in the countries participating in the study. The assistance of the Irish Cattle Breeding Federation in providing data relating to the Irish CEP is gratefully acknowledged. Scotland's Rural College (SRUC) gratefully acknowledges Helen Carty (SRUC Vet Services), Jenny Purcell, Ian Murdoch, and Paul Gavin (Scottish Government), the SRUC Beef \& Sheep KTE Group, and other colleagues at the Epidemiology Research Unit (ERU) for providing information relating to the Scottish CEP. SRUC also acknowledges the Scottish Government for provision of funding to ERU team members under the Strategic Research Program 20162021 Research Deliverables 2.2.6 Animal Disease Epidemiology. Also gratefully acknowledged is GDS Brittany for providing information on the CEP that runs in Brittany. We thank Mia Holmberg (SVA, Uppsala, Sweden) for her support in data collection. The authors have not stated any conflicts of interest.

\section{REFERENCES}

Cameron, A. R. 2012. The consequences of risk-based surveillance: Developing output-based standards for surveillance to demonstrate freedom from disease. Prev. Vet. Med. 105:280-286. https://doi .org/10.1016/j.prevetmed.2012.01.009.

Comin, A., B. Haesler, L. Hoinville, M. Peyre, F. Dorea, B. Schauer, L. Snow, K. Stärk, A. Lindberg, A. Brouwer, G. van Schaik, C. Staubach, K. Schulz, B. Bisdorff, F. Goutard, J. Pinto Ferreira, F. Conraths, A. Cameron, M. Martinez Aviles, J. Sanchez-Vizcaino, V. Varan, D. Traon, J. Pinto, J. Rushton, J. Ripperger, and D. Pfeiffer. 2016. RISKSUR Tools: Taking animal health surveillance into the future through interdisciplinary integration of scientific evidence. Pages 243-254 in Proc. Soc. Vet. Epidemiol. Prev. Med., Elsinore, Denmark. SVEPM, Elsinore, Denmark.

Fux, R., and G. Wolf. 2012. Transient elimination of circulating bovine viral diarrhoea virus by colostral antibodies in persistently infected calves: A pitfall for BVDV-eradication programs? Vet. Microbiol. 161:13-19. https://doi.org/10.1016/j.vetmic.2012.07.001.

Geraghty, T., D. A. Graham, P. Mullowney, and S. J. More. 2014. A review of bovine Johne's disease control activities in 6 endemically infected countries. Prev. Vet. Med. 116:1-11. https://doi.org/10 $.1016 /$ j.prevetmed.2014.06.003.

Graham, D. A., M. Lynch, S. Coughlan, M. L. Doherty, R. O'neill, D. Sammin, and J. O'flaherty. 2014. Development and review of the voluntary phase of a national BVD eradication programme in Ireland. Vet. Rec. 174:67. https://doi.org/10.1136/vr.101814.
Greiser-Wilke, I., B. Grummer, and V. Moennig. 2003. Bovine viral diarrhoea eradication and control programmes in Europe. Biologicals 31:113-118. https://doi.org/10.1016/s1045-1056(03)00025-3.

Heffernan, C., F. Misturelli, L. Nielsen, G. J. Gunn, and J. Yu. 2009. Analysis of pan-European attitudes to the eradication and control of bovine viral diarrhoea. Vet. Rec. 164:163-167. https://doi.org/ 10.1136/vr.164.6.163.

Hoinville, L. J., L. Alban, J. A. Drewe, J. C. Gibbens, L. Gustafson, B. Häsler, C. Saegerman, M. Salman, and K. D. C. Stärk. 2013. Proposed terms and concepts for describing and evaluating animalhealth surveillance systems. Prev. Vet. Med. 112:1-12. https://doi .org/10.1016/j.prevetmed.2013.06.006.

Houe, H. 2003. Economic impact of BVDV infection in dairies. Biologicals 31:137-143. https://doi.org/10.1016/s1045-1056(03)00030-7.

Houe, H., A. Lindberg, and V. Moennig. 2006. Test strategies in bovine viral diarrhea virus control and eradication campaigns in Europe. J. Vet. Diagn. Invest. 18:427-436. https://doi.org/10.1177/ 104063870601800501.

Hult, L., and A. Lindberg. 2005. Experiences from BVDV control in Sweden. Prev. Vet. Med. 72:143-148. https://doi.org/10.1016/j .prevetmed.2005.04.005.

Joly, A., C. Fourichon, and F. Beaudeau. 2005. Description and first results of a BVDV control scheme in Brittany (western France). Prev. Vet. Med. 72:209-213. https://doi.org/10.1016/j.prevetmed .2005.07.016.

Lindberg, A., and H. Houe. 2005. Characteristics in the epidemiology of bovine viral diarrhea virus (BVDV) of relevance to control. Prev. Vet. Med. 72:55-73. https://doi.org/10.1016/j.prevetmed 2005.07.018.

Mars, M. H., and C. van Maanen. 2005. Diagnostic assays applied in BVDV control in The Netherlands. Prev. Vet. Med. 72:43-48. https://doi.org/10.1016/j.prevetmed.2005.08.005.

Martin, P. A. J., A. R. Cameron, and M. Greiner. 2007. Demonstrating freedom from disease using multiple complex data sources: 1: A new methodology based on scenario trees. Prev. Vet. Med. 79:71-97. https://doi.org/10.1016/j.prevetmed.2006.09.008.

Moennig, V., H. Houe, and A. Lindberg. 2005. BVD control in Europe: Current status and perspectives. Anim. Health Res. Rev. 6:63-74. https://doi.org/10.1079/AHR2005102.

More, S. J., A. R. Cameron, M. Greiner, R. S. Clifton-Hadley, S. C. Rodeia, D. Bakker, M. D. Salman, J. M. Sharp, F. De Massis, A. Aranaz, M. B. Boniotti, A. Gaffuri, P. Have, D. Verloo, M. Woodford, and M. Wierup. 2009. Defining output-based standards to achieve and maintain tuberculosis freedom in farmed deer, with reference to member states of the European Union. Prev. Vet. Med. 90:254-267. https://doi.org/10.1016/j.prevetmed.2009.03 .013 .

National Veterinary Institute (SVA). 2015. Surveillance of infectious diseases in animals and humans in Sweden. SVA rapportserie 34. SVA, Uppsala, Sweden.

Olafson, P., and C. G. Rickard. 1947. Further observations on the virus diarrhea (new transmissible disease) of cattle. Cornell Vet. 37:104-106.

Pinior, B., C. L. Firth, V. Richter, K. Lebl, M. Trauffler, M. Dzieciol, S. E. Hutter, J. Burgstaller, W. Obritzhauser, P. Winter, and A. Käsbohrer. 2017. A systematic review of financial and economic assessments of bovine viral diarrhea virus (BVDV) prevention and mitigation activities worldwide. Prev. Vet. Med. 137:77-92. https: //doi.org/10.1016/j.prevetmed.2016.12.014.

Sandvik, T. 2004. Progress of control and prevention programs for bovine viral diarrhea virus in Europe. Vet. Clin. North Am. Food Anim. Pract. 20:151-169. https://doi.org/10.1016/j.cvfa.2003.12 .004 .

Schuppers, M. E., J. A. Stegeman, J. A. Kramps, and K. D. C. Stärk. 2012. Implementing a probabilistic definition of freedom from infection to facilitate trade of livestock: Putting theory into praxis for the example of bovine herpes virus-1. Prev. Vet. Med. 105:195201. https://doi.org/10.1016/j.prevetmed.2011.12.013.

Scottish Government. 2016. The Scottish BVD Eradication Scheme. Accessed Nov. 29, 2018. https://www2.gov.scot/Topics/ 
farmingrural/Agriculture/animal-welfare/Diseases/disease/bvd/ eradication.

The RISKSUR Project. 2015. RISKSUR: Risk-based animal health surveillance. Accessed Aug. 3, 2018. www.fp7-risksur.eu.

Toftaker, I., E. Ågren, M. Stokstad, A. Nødtvedt, and J. Frössling. 2018. Herd level estimation of probability of disease freedom applied on the Norwegian control program for bovine respiratory syncytial virus and bovine coronavirus. Prev. Vet. Med. https:// doi.org/10.1016/j.prevetmed.2018.07.002. In press.

van Duijn, L., A. M. B. Veldhuis, M. H. Mars, B. de Roo, and T. J. G. M. Lam. 2019. Efficacy of a voluntary BVDV control programme: Experiences from the Netherlands. Vet. J. 245:55-60. https://doi .org/10.1016/j.tvjl.2018.12.016. van Roon, A. M., I. M. G. A. Santman-Berends, D. Graham, S. J. More, M. Nielen, A. Madouasse, M. Mercat, C. Fourichon, J. Gethmann, J. Frössling, A. Lindberg, C. Correia-Gomes, G. J. Gunn, C. Sauter-Louis, M. K. Henry, L. van Duijn, and G. van Schaik. 2019. STOC Free: An innovative framework to compare probability of freedom from infection in heterogeneous control programmes. Front. Vet. Sci. 6:133. https://doi.org/10.3389/fvets .2019 .00133

Wernike, K., J. Gethmann, H. Schirrmeier, R. Schröder, F. J. Conraths, and M. Beer. 2017. Six years (2011-2016) of mandatory nationwide bovine viral diarrhea control in Germany-A success story. Pathogens 6:50. https://doi.org/10.3390/pathogens6040050. 